Original Paper http://ajol.info/index.php/ijbcs http://indexmedicus.afro.who.int

\title{
Effets du pâturage sur la biomasse herbacée et sur des paramètres chimiques et biologiques des sols dans une savane arbustive au Burkina Faso
}

\author{
Lambiénou YE ${ }^{1,2^{*}}$, Jean-Christophe LATA ${ }^{3,4}$, Dominique MASSE ${ }^{5}$, \\ Hassan Bismarck NACRO ${ }^{2}$ et Sébastien BAROT ${ }^{6}$ \\ ${ }^{1}$ Centre Universitaire Polytechnique de Dédougou, Université Ouaga I Pr Joseph KI-ZERBO, Burkina Faso \\ ${ }^{2}$ LERF, IDR, Université Polytechnique de Bobo-Dioulasso, Burkina Faso. \\ ${ }^{3}$ Sorbonne Universités, UPMC Univ Paris 06, IEES-Paris, 7 quai Saint Bernard, 75005 Paris, France. \\ ${ }^{4}$ Department of Geoecology and Geochemistry, Institute of Natural Resources, Tomsk Polytechnic University, \\ 30, Lenin Street, Tomsk 634050, Russia. \\ ${ }^{5}$ IRD, UMR 210 ECO\&SOLS (Montpellier SupAgro CIRAD INRA IRD), LMI IESOL (ISRA-UCAD-UT-IO- \\ INERA-IRD), place Viala (Bt. 12), F-34060 Montpellier cedex 2, France. \\ ${ }^{6}$ IRD, IEES-Paris, 7 quai Saint Bernard, 75005 Paris, France. \\ *Auteur correspondant, E-mail: ylambienou@yahoo.fr
}

\section{RESUME}

Cette étude a porté sur les sols et la végétation herbacée d'une savane pâturée au Burkina Faso. Elle décrit les effets du pâturage sur la biomasse herbacée et sur les paramètres chimiques et biologiques des sols. Pour ce faire, des couples de parcelles (protégées et non protégées du pâturage) ont été installés dans les faciès des quatre espèces de graminées dominantes: deux pérennes (A. ascinodis, A. gayanus) et deux annuelles (A. pseudapricus et L. togoensis) afin de tester les effets du pâturage. En plus de la mesure de la biomasse végétale, une évaluation des caractéristiques chimiques et biologiques des sols a aussi été effectuée. $\mathrm{Les}_{\mathrm{pH}}$ eau et $\mathrm{pH}_{\mathrm{KCl}}$, l'ammonium, le nitrate, la respiration basale, la bêta-glucosidase et la fluorescéine di-acétate des sols sont plus élevées sous les pérennes (A. ascinodis et A. gayanus), que sous les annuelles (A. pseudapricus et L. togoensis) et le sol nu. Le carbone total, l'azote total, le phosphore assimilable, le $\mathrm{C} / \mathrm{N}$, la biomasse microbienne et la phosphatase acide sont plus élevés sous la pérenne $A$. ascinodis que sous les autres espèces et le sol nu. Les résultats indiquent que le pâturage diminue significativement la biomasse aérienne sans affecter la biomasse racinaire. Par ailleurs, il n'y a pas d'effet significatif sur les paramètres chimiques et biologiques, excepté pour la respiration basale du sol qui augmente significativement en situation de non pâturage. On a donc obtenu en 18 mois d'exclos peu d'effets sur le fonctionnement du sol mais d'importants effets sur les biomasses herbacées.

() 2016 International Formulae Group. All rights reserved.

Mots clés : Graminées, herbivore, pâturage, caractéristiques chimiques et biologiques, savane.

\section{Effects of grazing on herbaceous biomass and chemical and biological parameters of soils in a shrub savanna in Burkina Faso}




\section{ABSTRACT}

This study focuses on the soils and herbaceous vegetation of a grazed savanna in Burkina Faso. It describes the effects of grazing on herbaceous biomass and soil chemical and biological parameters. Pairs of plots (protected from grazing and unprotected) were installed under the four dominant grass species $(A$. ascinodis, A. gayanus, A. pseudapricus and L. togoensis). In addition to the measure of plant biomass, an assessment of soil chemical and biological characteristics was also performed. $\mathrm{pH}_{\text {water }}$ and $\mathrm{pH}_{\mathrm{KCl}}$, ammonium, nitrate, basal respiration, $\beta$-glucosidase and fluorescein diacetate of soils are higher under perennial grasses $(A$. ascinodis and A. gayanus), than under annual species (A. pseudapricus and L. togoensis) and bare soil. Total carbon, total nitrogen, available phosphorus, $\mathrm{C} / \mathrm{N}$, microbial biomass, acid phosphatase of soil are higher under the perennial grass A. ascinodis than under the other species of grasses and bare soil. Results indicate that grazing decreases significantly herbaceous aboveground biomass (but not root biomass). However, grazing does not impact soil chemical and biological parameters, except for soil basal respiration that increases significantly in grazing situation. Thus, after 18 months of exclosure, we detect very little effect of cattle on soil functioning but important effects on the herbaceous biomass.

(c) 2016 International Formulae Group. All rights reserved.

Keywords: Grasses, herbivore, grazing, chemical and biological characteristics, Savanna.

\section{INTRODUCTION}

Dans les pays tropicaux, particulièrement au Burkina Faso, les sols et leur fertilité représentent une ressource précieuse. La gestion durable de cette ressource est donc une priorité pour nourrir les populations humaines de ces pays qui connaissent encore de fortes croissances démographiques. Les problèmes de fertilité des sols sont particulièrement aigus en Afrique notamment en zones sahélienne et soudanienne, où des précipitations plus faibles et réparties très inégalement au long de l'année rajoutent une contrainte supplémentaire pour la production végétale et l'évolution des sols. Dans ces zones l'agriculture dépend très étroitement de la fertilité des sols mais c'est aussi le cas de l'élevage qui reste essentiellement basé sur les pâturages naturels que constituent les savanes. Différents travaux ont montré que les herbivores, à travers le prélèvement de la biomasse végétale, le piétinement du sol et le dépôt d'urine et de fèces, ont d'importants effets sur les caractéristiques de la végétation (Fournier et al., 2001 ; Zoffoun et al., 2013) et sur le sol et son fonctionnement (Silveira et al., 2013).

Les herbivores, dans les écosystèmes naturels ou dans le cadre de l'élevage, affectent le recyclage des nutriments (Wardle et al., 2001) en interaction avec les espèces végétales, modifiant indirectement la qualité et la quantité de la biomasse végétale. La défoliation des plantes par les herbivores affecte non seulement la distribution, la biomasse et l'activité racinaire, mais aussi le rapport biomasse racinaire/biomasse aérienne (Guitian et Bardgett, 2000). La biomasse racinaire diminue ainsi généralement avec l'intensité de pâturage (Han et al., 2008). La modification du système racinaire des espèces végétales due à l'exploitation par les herbivores de la biomasse épigée, peut également stimuler la minéralisation de la matière organique du sol et les pertes de carbone associées (Han et al., 2008; Klumpp et al., 2009). Par ailleurs, les herbivores peuvent modifier directement (apport de fèces et d'urine et piétinement) ou indirectement (en modifiant la communauté et le fonctionnement des espèces végétales) les communautés et les activités microbiennes du sol (Patra et al., 2005; Klumpp et al., 2009) pouvant conduire à des effets négatifs ou positifs sur la minéralisation du carbone et de l'azote (Bardgett et Wardle, 2003). Ils influencent ainsi le stockage du carbone et de l'azote dans le sol qui diminue avec l'intensité de pâturage (Han et al., 2008). Les herbivores 
accélèrent le recyclage des nutriments, en particulier l'azote, par l'apport de matières organiques sous formes de fèces et d'urine qui se décomposent facilement (Le Roux et al., 2003; Patra et al., 2005). Le pâturage intensif stimulerait le taux de nitrification dans le sol alors que le pâturage léger stimulerait la dénitrification (Le Roux et al., 2003).

Tout ceci montre que les herbivores affectent le recyclage des nutriments par divers mécanismes. Dans le contexte de l'élevage, il est important de mieux analyser les interactions entre les différents processus conduisant à une modification du stockage et du recyclage des nutriments par les plantes en interaction avec le bétail. Ces interactions vont en effet déterminer la disponibilité des nutriments sur le long terme et donc influencer la diversité végétale et la durabilité de l'activité d'élevage. Lorsqu'elles améliorent l'efficacité du recyclage des nutriments, les modifications du recyclage des nutriments induites par l'herbivore peuvent entrainer une augmentation de la production primaire des écosystèmes (Boudsocq et al., 2009) mais on ne sait pas prédire dans quelles situations cela devrait arriver.

Dans les savanes exploitées par le bétail, on assiste fréquemment à la dégradation des pâturages due au surpâturage et en particulier via la dégradation de la végétation (Wittig et al., 2002). En effet, une mauvaise gestion des ressources fourragères peut engendrer des conséquences diverses. Afin de mettre en exergue l'importance du rôle du bétail dans la productivité et la durabilité des pâturages naturels, il s'avère donc nécessaire de déterminer et de comprendre ses effets sur les sols et la végétation. Au Burkina Faso, les études réalisées (Sawadogo et al., 2005) ont concerné surtout les effets du bétail sur la végétation, mais pas sur le recyclage des nutriments dans les savanes pâturées. De ce fait, l'objectif de ce travail est de mettre en évidence les effets $\mathrm{du}$ pâturage sur les paramètres chimiques et biologiques clés des sols sous des espèces de graminées par la mise en place d'exclos dans une savane pâturée dans la zone de BoboDioulasso au Burkina Faso. La connaissance de ces effets devrait permettre, en partie, de développer des stratégies de gestion durable de ces écosystèmes (savanes) exploités par le bétail. Il s'est agi pour nous de déterminer le $\mathrm{pH}$ et les teneurs en carbone total, en azote total, en phosphore assimilable, en ammonium et en nitrate des sols sous les deux espèces dominantes de graminées pérennes (Andropogon ascinodis et Andropogon gayanus) et sous les deux espèces dominantes de Graminées annuelles (Andropogon pseudapricus et Loudetia togoensis) dans des couples de parcelles (protégées et non protégées du pâturage).

Les paramètres biologiques (biomasse microbienne; activités enzymatiques, etc.) jouent également un rôle clé dans le fonctionnement des sols. En effet, ils influencent le stockage, la disponibilité et le recyclage des nutriments. Du fait de leur lien étroit avec les cycles biogéochimiques des nutriments, les paramètres biologiques notamment la biomasse microbienne (Groffman et al., 2001) et les activités enzymatiques (Gonnety et al., 2012) sont très souvent mesurés pour juger de la qualité des sols. Pour ce faire, nous avons donc mesuré la respiration basale, la biomasse microbienne et les activités enzymatiques de la bêtaglucosidase, de la fluorescéine di-acétate et de la phosphatase acide.

Pour atteindre notre objectif, nous avons émis les hypothèses suivantes :

Le bétail modifie les activités biologiques des sols ;

Le pâturage impacte le stockage des nutriments dans le sol.

\section{MATERIEL ET METHODES}

\section{Site d'étude}

Le site d'étude est l'unité d'aménagement $n^{\circ} 3$ de la Forêt Classée de Dindérésso située à l'Ouest de la ville de Bobo-Dioulasso $\left(11^{\circ} 12.494\right.$ ' de latitude Nord, $4^{\circ} 24.159^{\prime}$ de longitude Ouest et $390 \mathrm{~m}$ d'altitude) (Yé et al., 2015). Le climat est de 
type sud-soudanien caractérisé par deux saisons : une saison humide de mai à octobre et une saison sèche de novembre à avril. La zone est située entre les isohyètes $900 \mathrm{~mm}$ et $1200 \mathrm{~mm}$. Les quantités moyennes d'eau de pluies enregistrées pour les trois années d'observations sont: 1289,5 mm en 2010 ; $831 \mathrm{~mm}$ en 2011 et $1089 \mathrm{~mm}$ en 2012. La température moyenne annuelle est de $28{ }^{\circ} \mathrm{C}$.

Les sols appartiennent aux groupes de sols ferrugineux tropicaux lessivés modaux et ferrugineux tropicaux lessivés indurés. Le site présente des faciès à dominance d'herbacées annuelles et des faciès à dominance d'herbacées pérennes. Le pâturage est plus ou moins homogène sur tout le site en saison sèche. Ce site connait également un passage régulier annuel des feux de brousse. De ce fait, il a été subdivisé en quatre blocs en fonction des faciès à dominance d'herbacées annuelles ou pérennes d'une part et d'autre part, en fonction de la fréquentation du bétail. . Les blocs 1 et 2 sont dominés par les faciès de graminées annuelles (A. pseudapricus et $L$. togoensis) et sont plus fréquentés par le bétail en saison pluvieuse tandis que les blocs 3 et 4 sont dominés par les faciès de graminées pérennes (A. ascinodis et $A$. gayanus) et sont moins fréquentés par le bétail en saison des pluies. Dans les blocs 1 et 2, les sols sont peu ou moyennement profonds (profondeur $\leq 55$ $\mathrm{cm}$ ). Par contre, dans les blocs 3 et 4 , les sols sont profonds (profondeur $\geq 105 \mathrm{~cm}$ ).

C'est une savane arbustive pâturée constituée des espèces ligneuses telles que Vitellaria paradoxa, Terminalia laxiflora, Detarium microcarpum, Parkia biglobosa, Guiera senegalensis, Combretum nigricans, Gardenia ternifolia, etc. Les espèces herbacées sont Andropogon gayanus, Loudetia togoensis, Indigofera trichopoda, Andropogon ascinodis, Andropogon pseudapricus, Hyparrhenia subplumosa, Aristida kerstingii, Cassia mimosoides, Microchloa indica, etc.

\section{Comparaison des effets du pâturage}

En vue de déterminer les effets du bétail sur les caractéristiques de la strate herbacée et sur les sols, un dispositif expérimental comportant des couples de parcelles a donc été mis en place à la fin du mois de mai 2011 afin de tester les effets pression du pâturage. Dans chaque bloc, il y a deux espèces de graminées (annuelles ou pérennes) dominantes. De ce fait, trois couples de parcelles ont été mis en place de façon aléatoire par faciès des deux graminées dominantes. Chaque couple de parcelle comporte une parcelle protégée du pâturage à l'aide d'une clôture en fil barbelé et une parcelle non protégée ayant chacune $16 \mathrm{~m}^{2}$ et distantes de $3 \mathrm{~m}$ l'une de l'autre. Par bloc il y a donc 6 couples de parcelles soit au total 24 couples (48 parcelles) pour les quatre blocs. Après 18 mois (octobre 2012) de manipulation, des échantillons de sols ont été prélevés dans chaque parcelle et la biomasse végétale herbacée des différentes parcelles a été également estimée.

\section{Mesure de la biomasse herbacée}

La méthode de la récolte intégrale a été utilisée et a été effectuée sur les 48 parcelles. La récolte a été réalisée sur cinq placettes d'un mètre carré disposées suivant les deux médianes à l'intérieur de chaque parcelle. La biomasse fraîche a été pesée immédiatement sur le terrain et ensuite un échantillon composite a été prélevé pour l'évaluation de la biomasse sèche après séchage, jusqu'à poids constant, à l'étuve. Concernant la biomasse racinaire, des carottes de sols ont été prélevées à l'aide d'une tarière de $5 \mathrm{~cm}$ de diamètre sur l'horizon 0-10 cm sur les placettes ayant servies à l'estimation de la biomasse aérienne dans chaque parcelle. Les racines ont été extraites par lavage à l'eau du robinet en utilisant un tamis. Elles ont ensuite été séchées jusqu'à poids constant à l'étuve puis pesées pour la détermination du poids sec.

\section{Prélèvement et échantillonnage des sols}

Dans chaque parcelle, des échantillons de sol ont été prélevés, à l'aide d'une tarière, 
sous des individus $(\mathrm{n}=5)$, choisis aléatoirement, de la graminée dominante et sur des sols nus $(n=5)$ situés entre les touffes d'herbes sur l'horizon $0-10 \mathrm{~cm}$. Les cinq échantillons élémentaires prélevés sous les cinq individus de l'espèce dominante ont ensuite été regroupés pour former un échantillon composite. De même pour le sol nu, les échantillons ont été regroupés pour constituer des échantillons composites. Cela conduit donc à deux échantillons composites par parcelle et au total 96 échantillons composites pour les 48 parcelles. Ces échantillons de sols ont été séchés à l'air ambiant à l'ombre, tamisés à $2 \mathrm{~mm}$ puis conditionnés dans des sachets plastiques avant les différentes analyses.

\section{Analyse des sols au laboratoire}

Le carbone total et l'azote total ont été déterminés par CHN (analyseur élémentaire, Thermo Funnigan Flash EA 1112). Le phosphore assimilable a été déterminé suivant la méthode Olsen modifiée Dabin (Dabin, 1967).

Les $\mathrm{pH}_{\text {eau }}$ et $\mathrm{pH}_{\mathrm{KCl}}$ ont été mesurés par la méthode électrométrique au $\mathrm{pH}$ mètre à électrodes en verre au laboratoire LAMAIRD-Dakar. La solution utilisée pour la lecture $\mathrm{du} \mathrm{pH}_{\text {eau }}$ a été préparée dans un rapport échantillon/eau de 1/2,5 (10g de sol + $25 \mathrm{ml}$ d'eau). A cette solution, on a ajouté ensuite $1,86 \mathrm{~g}$ de $\mathrm{KCl}$ afin de déterminer le $\mathrm{pH}_{\mathrm{KCl}}$. Ces mesures ont été effectuées suivant le protocole du LAMA-IRD-Dakar au Sénégal (laboratoire certifié ISO 9001)

La respiration basale du sol reflète l'activité respiratoire des microorganismes du sol qui en minéralisant la matière organique produisent du $\mathrm{CO}_{2}$. Le principe est basé sur la mesure du dégagement de $\mathrm{CO}_{2}$ par rapport au volume total d'air en fonction du temps, par l'échantillon de sol incubé dans une enceinte close. Les échantillons de sols sont donc humidifiés à $80 \%$ de leur capacité de rétention dans des flacons en verre de $250 \mathrm{ml}$, bien homogénéisés puis mis en incubation à $28{ }^{\circ} \mathrm{C}$. Les mesures du $\mathrm{CO}_{2}$ dégagé sont effectuées une fois toutes les 24 heures pendant sept jours à partir du temps initial T0 par micro chromatographie en phase gazeuse MTI 200. Ces sols incubés pendant une semaine pour la respiration basale, ont été utilisés ensuite pour la détermination de la biomasse microbienne, pour l'extraction de l'ammonium et des nitrates et pour l'estimation des activités enzymatiques.

La biomasse microbienne a été déterminée par la méthode de fumigationextraction. Le principe est basé sur le dosage de l'azote $\alpha$-aminé contenu dans les microorganismes obtenu par extraction au $\mathrm{KCl}$, après incubation dans un milieu saturé en chloroforme (= fumigation) destiné à tuer les organismes. La quantité de carbone présente dans la biomasse microbienne est ensuite calculée en multipliant la quantité d'azote $\alpha$ aminé obtenu lors de l'incubation par le facteur 21 (Amato et Ladd, 1988) selon la formule suivante :

BM-C $=(\mathbf{N} \alpha$-aminé sol fumigé - $\mathbf{N} \alpha-$ aminé sol non fumigé)*21.

L'ammonium $\left(\mathrm{NH}_{4}{ }^{+}\right)$et les nitrates $\left(\mathrm{NO}_{3}{ }^{-}\right)$contenus dans les échantillons de sol sont extraits au $\mathrm{KCl} 1 \mathrm{M}$ puis dosés par colorimétrie avec un auto-analyseur à flux continu (Bremner, 1965).

Les activités de la bêta-glucosidase, de la fluorescéine di-acétate et de la phosphatase acide ont été déterminées au LEMSAT/IRDDakar. Pour la bêta-glucosidase (Hayano, 1973), on prend $0,1 \mathrm{~g}$ de sol dans un tube à hémolyse et on ajoute $400 \mu \mathrm{l}$ d'une solution tampon (Tampon Citrate phosphate) à pH 5,8 plus $100 \mu \mathrm{l}$ de solution du substrat para-nitrophényl $\beta$-D-glucopyranoside (SIGMA N7006). L'ensemble est mis en incubation pendant $2 \mathrm{~h}$ à $37{ }^{\circ} \mathrm{C} \pm 1{ }^{\circ} \mathrm{C}$. La réaction est stoppée avec $3 \mathrm{ml}$ d'une solution de $\mathrm{Na}_{2} \mathrm{CO}_{3}$. La quantité de para-nitro-phénol (p-NP) libéré par l'activité de la bêta-glucosidase est mesurée au spectrophotomètre à une longueur d'onde de $400 \mathrm{~nm}$. Pour la mesure de la fluorescéine di-acétate (FDA) (Adam et Duncan, 2001), on pèse $1 \mathrm{~g}$ de sol dans un tube à essai opaque et on ajoute $15 \mathrm{ml}$ de la solution tampon (tampon potassium phosphate) à $\mathrm{pH} 7,6$ plus $200 \mu \mathrm{l}$ de substrat FDA (SIGMA F7378). L'ensemble est mis en incubation pendant $1 \mathrm{~h}$ à $30^{\circ} \mathrm{C} \pm 1{ }^{\circ} \mathrm{C}$. La 
réaction est arrêtée par $1 \mathrm{ml}$ d'acétone. La quantité de la fluorescéine libérée est mesurée au spectrophotomètre à une longueur d'onde de $490 \mathrm{~nm}$. Concernant la phosphatase acide (Tabatabai et Bremner, 1969), 0,1 g de sol pesé dans un tube à hémolyse est mis en incubation pendant $1 \mathrm{~h}$ à $37{ }^{\circ} \mathrm{C} \pm 1{ }^{\circ} \mathrm{C}$ avec $400 \mu \mathrm{l}$ de solution tampon (Tampon universel modifié) plus $100 \mu \mathrm{l}$ de substrat para-nitrophényl phosphate p-NPP (104-0 SIGMA phosphatase substrate). Au bout d'une heure d'incubation, on ajoute $100 \mu \mathrm{l}$ de solution de $\mathrm{CaCl}_{2}$ puis ensuite $400 \mu \mathrm{l}$ de $\mathrm{NaOH}$ pour arrêter la réaction. La quantité de para-nitrophénol (p-NP) libérée est mesurée au spectrophotomètre à la longueur d'onde de $400 \mathrm{~nm}$.

\section{Analyses statistiques des données}

Le traitement statistique des données et les figures ont été réalisés grâce au logiciel $\mathrm{R}$ version 2142 . Les effets des espèces de graminées et de la protection ont été testés en utilisant des modèles à effets mixtes avec le bloc comme effet aléatoire. La direction des effets et les modalités des traitements significativement différents des autres ont été déterminées par simplification des modèles en regroupant les modalités (Crawley, 2007).

\section{RESULTATS \\ Effets du pâturage sur la biomasse herbacée}

Les résultats des effets du pâturage sur la biomasse herbacée sont présentés par la Figure 1 et le Tableau 1. Les biomasses aérienne et racinaire varient significativement entre les différents faciès des quatre espèces de graminées (Tableau 1). Pour les parcelles protégées du bétail, les quantités moyennes de biomasse aérienne en tonne de matière sèche par hectare $\left(\mathrm{tMS} \mathrm{ha}{ }^{-1}\right)$ sont de 2,68; 3,06; 3,22 et 2,42 et pour les biomasses racinaires de 0,$34 ; 0,59 ; \quad 7,77$ et 3,16 tMS $\mathrm{ha}^{-1}$ respectivement pour $L$. togoensis, $A$. pseudapricus, A. ascinodis et A. gayanus. Pour les parcelles non protégées, les quantités moyennes de biomasses aériennes en tMS ha ${ }^{1}$ sont de 1,$62 ; 1,78 ; 1,97$ et 0,75 et pour les biomasses racinaires de 0,$27 ; 0,20 ; 6,37$ et
1,90 tMS $\mathrm{ha}^{-1}$ respectivement pour $L$. togoensis, A. pseudapricus, A. ascinodis et A. gayanus. Quelle que soit la situation (parcelle protégée ou non), le faciès à $A$. gayanus présente la biomasse aérienne la plus faible tandis que les quantités de biomasses racinaires les plus élevées sont obtenues dans les faciès à graminées pérennes $A$. ascinodis et A. gayanus (Figure 1). Dans chaque faciès de graminées, on note que les parcelles protégées présentent les quantités de biomasses aériennes les plus élevées (Figure 1). Cependant, il n'y a pas de différence significative entre les biomasses racinaires des deux types de parcelles (Tableau 1).

\section{Effets du pâturage sur les paramètres chimiques des sols}

Les résultats des effets sur les paramètres chimiques des sols sont représentés par les Figures 2, 3 et 4 et le Tableau 2. Ils montrent (Tableau 2) des différences significatives pour tous les paramètres chimiques entre les quatre espèces de graminées. Les valeurs des $\mathrm{pH}_{\text {eau }}$ et $\mathrm{pH}_{\mathrm{KCl}}$ sont plus élevées sous les pérennes $A$. ascinodis et $A$. gayanus, que pour le sol nu et les deux espèces annuelles $A$. pseudapricus et L. togoensis (Figure 2). Les teneurs en carbone total, en azote total, en phosphore assimilable et le rapport $\mathrm{C} / \mathrm{N}$ sont plus élevés sous $A$. ascinodis que sous les autres espèces de graminées et le sol nu (Figure 3 et 4). Les teneurs en ammonium et en nitrates sont plus élevées sous les pérennes que sous les annuelles et le sol nu (Figure 4). Pour tous les paramètres chimiques analysés, les résultats d'analyse (Tableau 2) montrent une absence de différences significatives entre les parcelles protégées et non protégées quelle que soit l'espèce de graminée.

\section{Effets du pâturage sur les paramètres biologiques des sols}

Les résultats des effets du pâturage sur les paramètres biologiques des sols sont présentés par les Figures 5 et 6 et le Tableau 3. Les résultats d'analyse (Tableau 3) montrent des différences signicatives pour tous les paramètres biologiques. La respiration 
basale (Figure 5) et les activités de la bêtaglucosidase et de la fluorescéine di-acétate (Figure 6) sont plus élevées sous les graminées pérennes $A$. ascinodis et $A$. gayanus, que sous le sol nu et les annuelles $A$. pseudapricus et L. togoensis. Quant à la biomasse microbienne (Figure 5) et la phosphatase acide (Figure 6), elles apparaissent plus élevées sous la pérenne $A$. ascinodis que sous les autres espèces et le sol nu. Les résultats révèlent également, pour chaque espèce de graminée, une différence significative entre les parcelles protégées et non protégées uniquement pour la respiration basale. Elle est plus élevée dans les parcelles protégées que dans les parcelles non protégées.
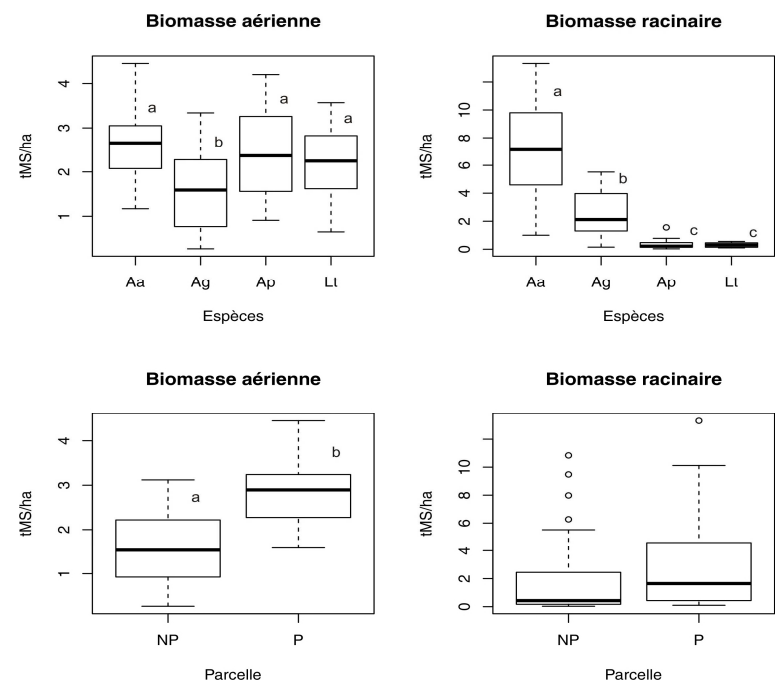

Figure 1 : variation de la biomasse herbacée en fonction des faciès de Graminées et de la protection. Aa (A. ascinodis), $\mathrm{Ag}$ (A. gayanus), $\mathrm{Ap}$ (A. pseudapricus) et $\mathrm{Lt}($ L. togoensis). $\mathrm{P}=$ parcelle protégée et $\mathrm{NP}=$ parcelle non protégée. tMS/ha (tonne de matière sèche par hectare). Les lettres $\mathrm{a}, \mathrm{b}$ et $\mathrm{c}$ représentent la significativité entre traitements. Les traitements affectés de la même lettre ne sont pas significativement différents.
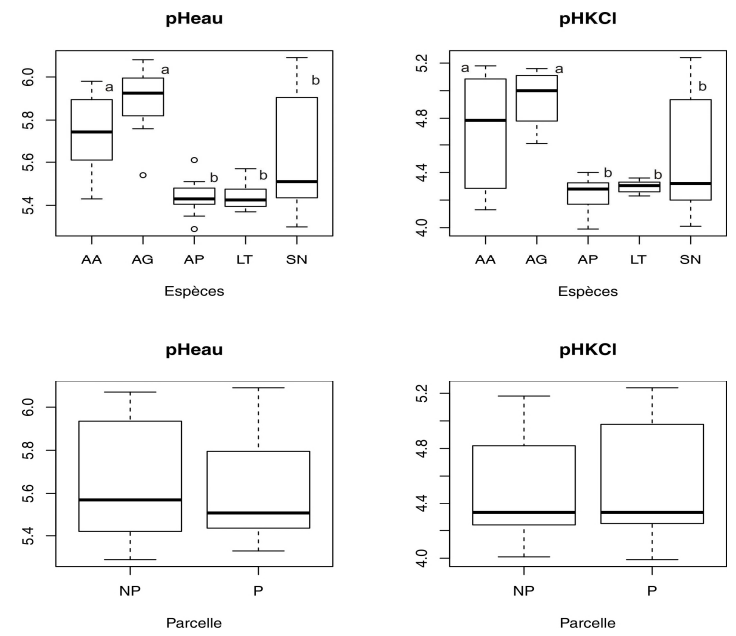

Figure 2 : variation du $\mathrm{pH}_{\text {eau }}$ et $\mathrm{pH}_{\mathrm{KCl}}$ des sols. 
$\mathrm{AA}($ A. ascinodis $) ; \mathrm{AG}($ A. gayanus $) ; \mathrm{AP}($ A. pseudapricus $) ; \mathrm{LT}($ L. togoensis $)$ et $\mathrm{SN}(\mathrm{Sol}$ nu $) . \mathrm{P}=$ parcelle protégée $; \mathrm{NP}=$ parcelle non protégée. Les lettres a et $\mathrm{b}$ représentent la significativité entre traitements. Les traitements portant la même lettre ne sont pas significativement différents.
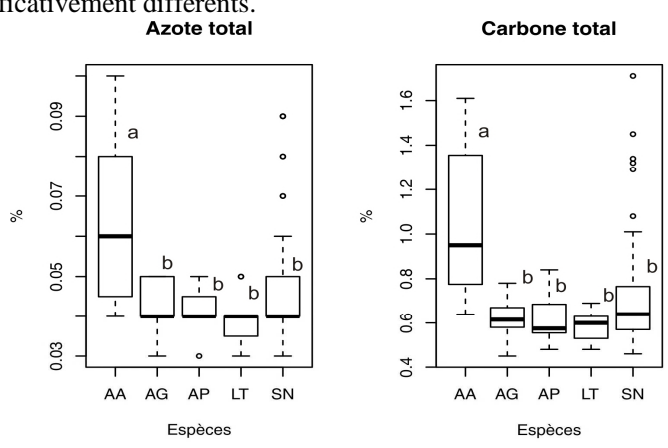

Rapport C/N
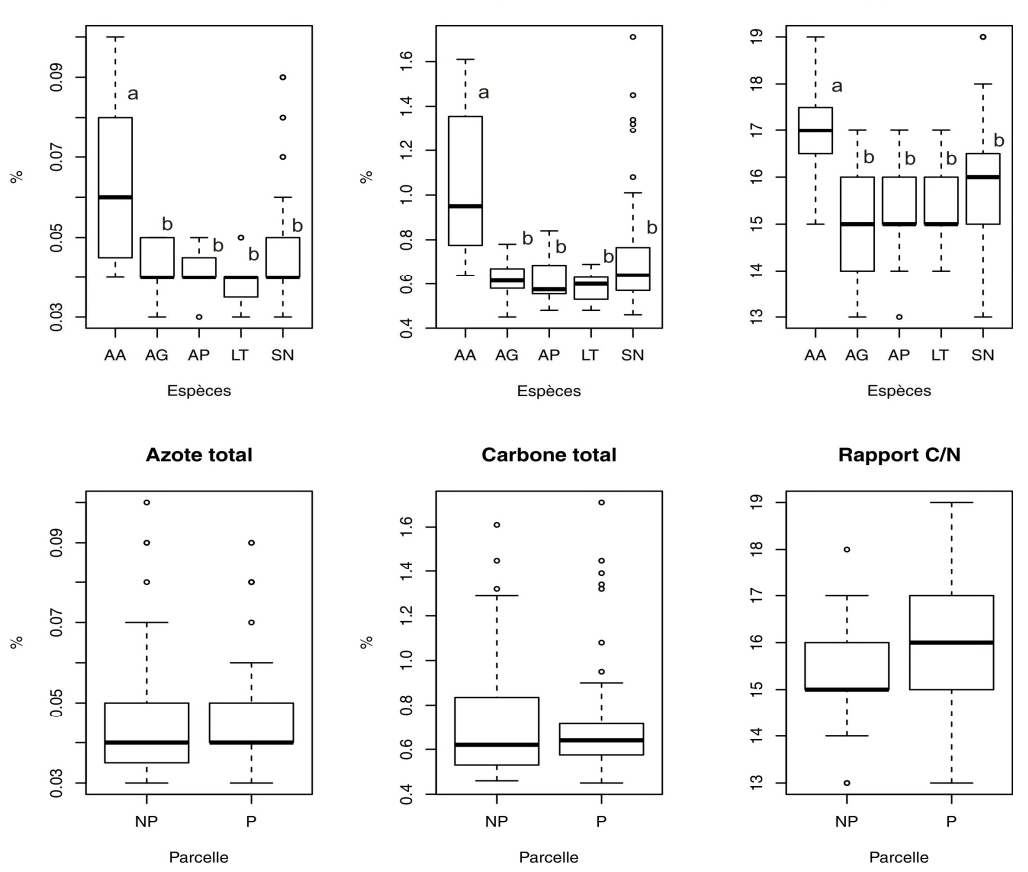

Figure 3 : variation des teneurs en carbone total et en azote des sols et le rapport $\mathrm{C} / \mathrm{N}$. $\mathrm{AA}$ (A. ascinodis) ; AG (A. gayanus) ; AP (A. pseudapricus) ; LT (L. togoensis) et SN (Sol nu). $\mathrm{P}=$ parcelle protégée ; $\mathrm{NP}=$ parcelle non protégée. Les lettres a et b représentent la significativité entre traitements. Les traitements portant la même lettre ne sont pas significativement différents.
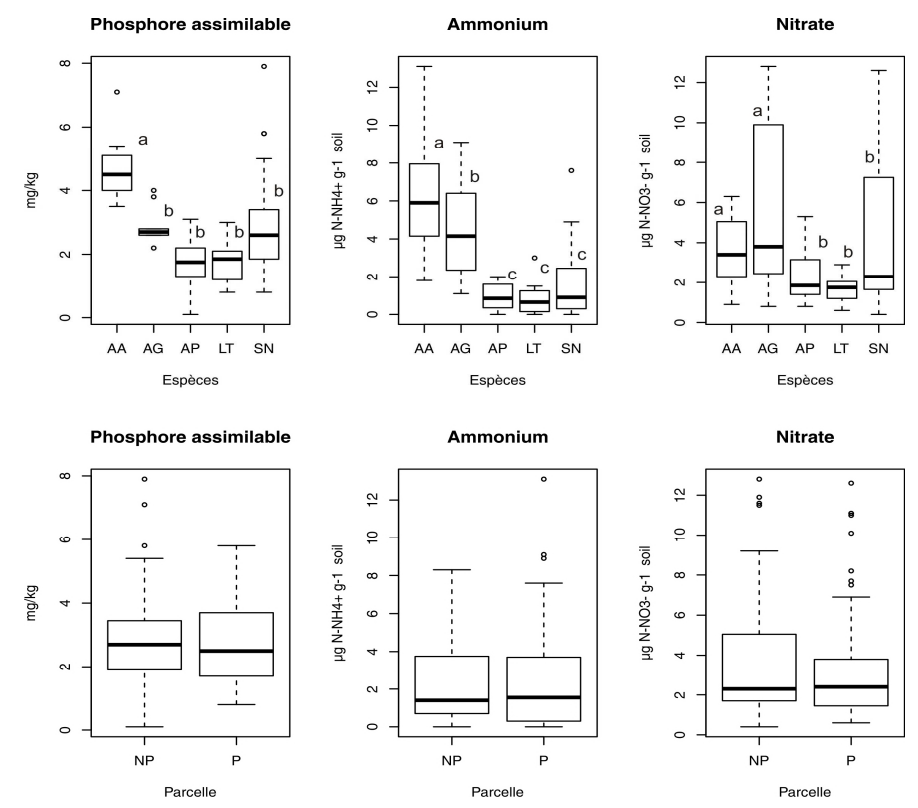

Figure 4 : variation des teneurs en phosphore assimilable, en ammonium et en nitrate des sols. 
$\mathrm{AA}$ (A. ascinodis) ; AG (A. gayanus) ; AP (A. pseudapricus) ; LT (L. togoensis) et $\mathrm{SN}$ ( $\mathrm{Sol}$ nu). $\mathrm{P}=$ parcelle protégée ; $\mathrm{NP}=$ parcelle non protégée. 1, 2, 3 et 4 sont les numéros des blocs. Les lettres a, b et c représentent la significativité entre traitements. Les traitements portant la même lettre ne sont pas significativement différents.
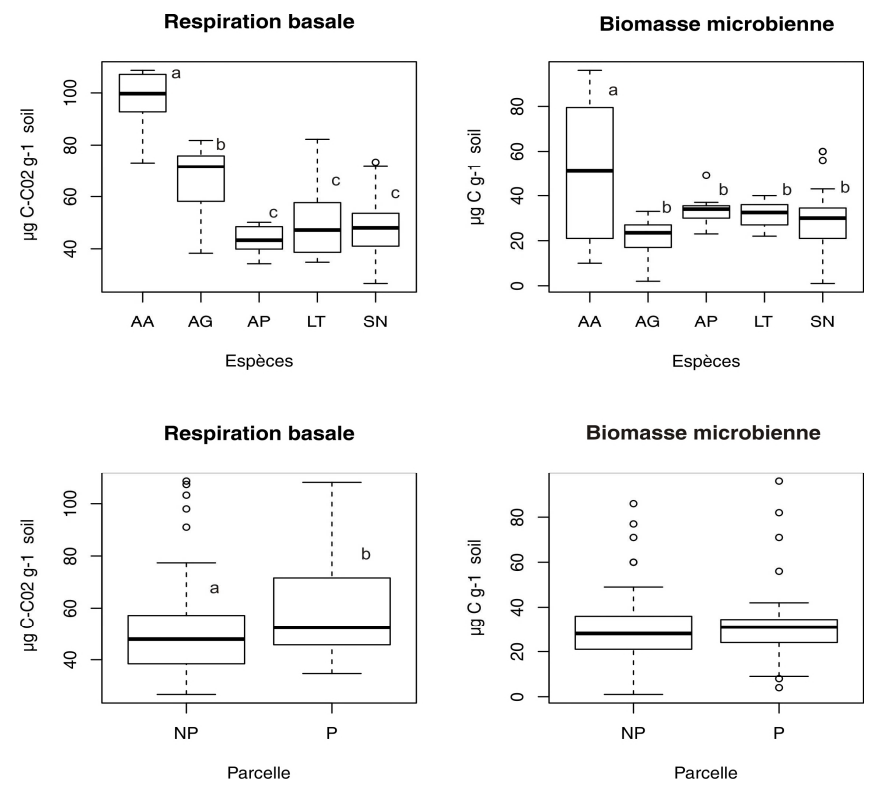

Figure 5 : variation de la biomasse microbienne et de la respiration basale des sols. AA (A. ascinodis) ; AG (A. gayanus) ; AP (A. pseudapricus) ; LT (L. togoensis) et SN (Sol nu). P = parcelle protégée ; $\mathrm{NP}=$ parcelle non protégée. Les lettres $\mathrm{a}, \mathrm{b}$ et $\mathrm{c}$ représentent la significativité entre traitements. Les traitements portant la même lettre ne sont pas significativement différents.
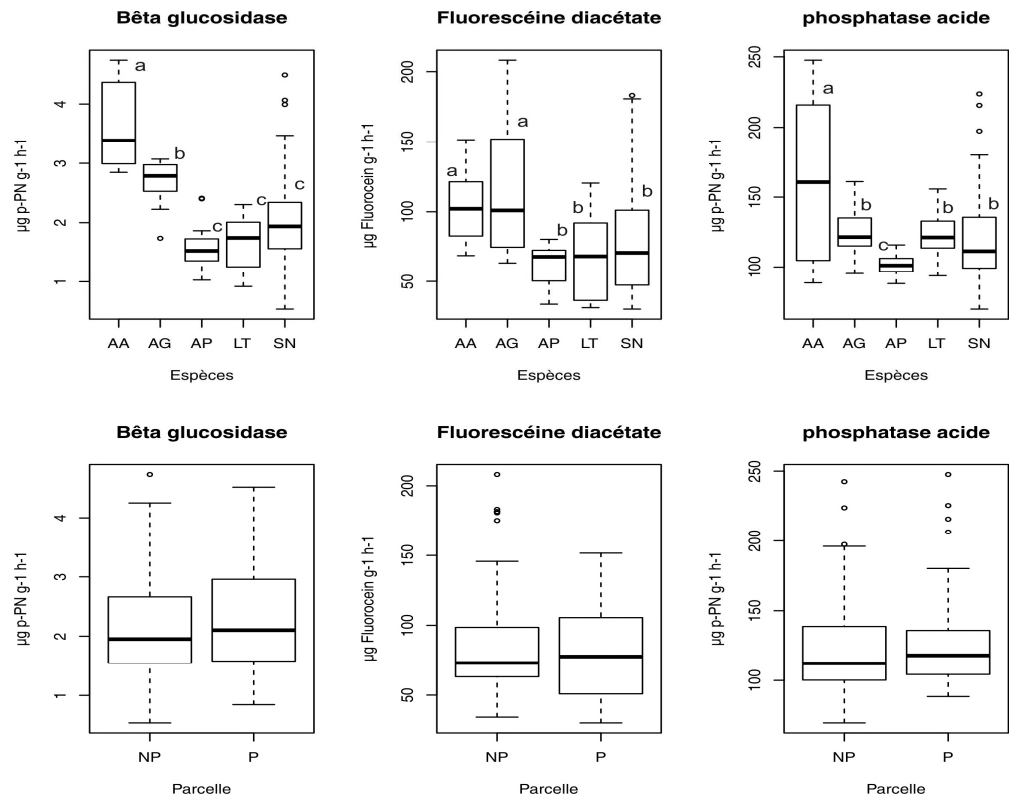

Figure 6 : variation des activités enzymatiques des sols. 
L. YE et al. / Int. J. Biol. Chem. Sci. 10(6): 2539-2554, 2016

AA (A. ascinodis) ; AG (A. gayanus) ; AP (A. pseudapricus) ; LT (L. togoensis) et $\mathrm{SN}$ (Sol nu). P = parcelle protégée ;

$\mathrm{NP}=$ parcelle non protégée. Les lettres $\mathrm{a}, \mathrm{b}$ et $\mathrm{c}$ représentent la significativité entre traitements. Les traitements portant la même lettre ne sont pas significativement différents. 
Tableau 1 : biomasse herbacée en fonction de la protection et des espèces de graminées.

\begin{tabular}{lccc}
\hline & DF & Biomasse aérienne & Biomasse racinaire \\
\hline Protection & 1 & $37,94^{* * *}$ & $1,33^{\text {ns }}$ \\
Espèce & 3 & $2,68^{*}$ & $17,19^{* * *}$ \\
Direction des effets & & $\mathrm{P}>\mathrm{NP}$ et & $\mathrm{Aa}>\mathrm{Ag}>\mathrm{Ap}, \mathrm{Lt}$
\end{tabular}

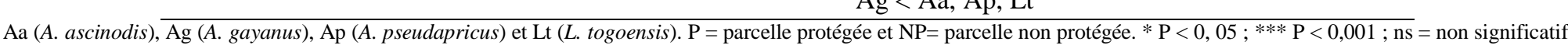

Tableau 2: paramètres chimiques des sols en fonction de la protection et des espèces de graminées.

\begin{tabular}{|c|c|c|c|c|c|c|c|c|c|}
\hline & DF & $\mathbf{p H}_{\text {eau }}$ & $\mathbf{p H}_{\mathrm{KCl}}$ & Azote total & Carbone total & $\mathbf{C} / \mathbf{N}$ & P. ass. & $\mathrm{NH}_{4}^{+}$ & $\mathrm{NO}_{3}^{-}$ \\
\hline Protection & 1 & $0,95^{\mathrm{ns}}$ & $0,32^{\mathrm{ns}}$ & $0,01^{\mathrm{ns}}$ & $0,11^{\mathrm{ns}}$ & $2,73^{\mathrm{ns}}$ & $0,73^{\text {ns }}$ & $0,15^{\mathrm{ns}}$ & $0,55^{\mathrm{ns}}$ \\
\hline $\begin{array}{l}\text { Espèce et } \\
\text { sol nu }\end{array}$ & 4 & $4,96 * * *$ & $2,36^{*}$ & $4,53 * *$ & $6,78 * * *$ & $4,40 * *$ & $5,11 * * *$ & $18,12 * * *$ & $3,04 *$ \\
\hline $\begin{array}{c}\text { Direction des } \\
\text { effets }\end{array}$ & & $\begin{array}{c}\mathrm{Aa}, \mathrm{Ag}>\mathrm{Ap}, \\
\mathrm{Lt}, \mathrm{SN}\end{array}$ & $\begin{array}{c}\mathrm{Aa}, \mathrm{Ag}> \\
\mathrm{Ap}, \mathrm{Lt}, \mathrm{SN}\end{array}$ & $\begin{array}{c}\mathrm{Aa}>\mathrm{Ag}, \mathrm{Ap}, \\
\mathrm{Lt}, \mathrm{SN}\end{array}$ & $\mathrm{Aa}>\mathrm{Ag}, \mathrm{Ap}, \mathrm{Lt}, \mathrm{SN}$ & $\begin{array}{c}\mathrm{Aa}>\underset{\mathrm{SN}}{\mathrm{Ag}, \mathrm{A}, \mathrm{Lt},} \\
\end{array}$ & $\begin{array}{c}\mathrm{Aa}>\mathrm{Ag}, \mathrm{Ap}, \mathrm{Lt}, \\
\mathrm{SN}\end{array}$ & $\begin{array}{l}\mathrm{Aa}>\mathrm{Ag}> \\
\mathrm{Ap}, \mathrm{Lt}, \mathrm{SN}\end{array}$ & $\begin{array}{c}\mathrm{Aa}, \mathrm{Ag}> \\
\mathrm{Ap}, \mathrm{Lt}, \mathrm{SN}\end{array}$ \\
\hline
\end{tabular}

$\mathrm{Aa}($ A. ascinodis $) ; \mathrm{Ag}($ A gayanus $) ; \mathrm{Ap}($ A. pseudapricus $) ; \mathrm{Lt}$ (L. togoensis) et SN (Sol nu).

$* \mathrm{P}<0,05 ; * * \mathrm{P}<0,01 ; * * * \mathrm{P}<0,001$ et ns $=$ non significatif.

Tableau 3: paramètres biologiques des sols en fonction de la protection et des espèces de raminées.

\begin{tabular}{|c|c|c|c|c|c|c|}
\hline & DF & Biomasse microbienne & Respiration basale & Bêta-glucosidase & Fluorescéine di acétate & Phosphatase acide \\
\hline Protection & 1 & $0,03^{\text {ns }}$ & $3,37 *$ & $2,26^{\mathrm{ns}}$ & $2,18^{\mathrm{ns}}$ & $0,79^{\mathrm{ns}}$ \\
\hline Espèce et sol nu & 4 & $8,91 * * *$ & $47,51 * * *$ & $7,91 * * *$ & $0,77 *$ & $4,55 * * *$ \\
\hline $\begin{array}{l}\text { Direction des } \\
\text { effets }\end{array}$ & & $\mathrm{Aa}>\mathrm{Ag}, \mathrm{Ap}, \mathrm{Lt}, \mathrm{SN}$ & $\mathrm{Aa}>\mathrm{Ag}>\mathrm{Ap}, \mathrm{Lt}, \mathrm{SN}$ & $\mathrm{Aa}>\mathrm{Ag}>\mathrm{Ap}, \mathrm{Lt}, \mathrm{SN}$ & $\mathrm{Aa}, \mathrm{Ag}>\mathrm{Ap}, \mathrm{Lt}, \mathrm{SN}$ & $\mathrm{Aa}>\mathrm{Ag}, \mathrm{Lt}>\mathrm{Ap}, \mathrm{SN}$ \\
\hline
\end{tabular}




\section{DISCUSSION}

Dans l'ensemble, les valeurs moyennes de la biomasse végétale du site, même pour les parcelles protégées où elles apparaissent élevées, restent relativement faibles par rapport à la biomasse d'autres savanes dans la sous-région (Fournier et al., 2001). Ceci pourrait être dû, en partie, à des différences entre les qualités des sols, la composition spécifique de la végétation herbacée, l'intensité d'exploitation de ces savanes et entre les périodes d'estimation de la biomasse.

Les biomasses aériennes et racinaires varient significativement en fonction des espèces de graminées. Cela est conforme aux résultats de certains auteurs (Sawadogo et al., 2005; Oumorou et al., 2010; Toko et Sinsin, 2011; Yaméogo et al., 2013) qui ont montré que la biomasse végétale herbacée dépend de la composition spécifique, du type de sol, du type de pâturage, etc. La biomasse racinaire des graminées pérennes est plus élevée. Ceci serait dû au fait que les graminées pérennes développent un système racinaire plus dense que les annuelles et produiraient ainsi une biomasse racinaire qui dépasse très souvent le double de leur biomasse aérienne (Fournier et al., 2001). Les résultats montrent que le pâturage affecte significativement la biomasse aérienne. Elle est plus élevée dans les parcelles protégées que dans les parcelles non protégées. Ces résultats montrent donc que le bétail entraine, comme il fallait s'y attendre, une diminution de la biomasse aérienne (Rakotoarimanana et al., 2008). Ces résultats montrent, par ailleurs, un effet non significatif $\mathrm{du}$ pâturage sur la biomasse racinaire. Cependant, le pâturage (pendant plusieurs années) entrainerait la diminution aussi bien de la biomasse aérienne que racinaire (Han et al., 2008). Ainsi ce résultat serait lié à la courte durée de l'expérimentation.

Les valeurs des $\mathrm{pH}_{\text {eau }}$ et $\mathrm{pH}_{\mathrm{KCl}}$ sont plus élevées sous les graminées pérennes $A$. ascinodis et A. gayanus, que sous le sol nu et les graminées annuelles $A$. pseudapricus et $L$. togoensis. Ces résultats indiqueraient donc que ces deux types de graminées influencent différemment le $\mathrm{pH}$ du sol suivant des mécanismes qui restent à déterminer. En effet, les espèces végétales peuvent modifier le $\mathrm{pH}$ du sol, surtout de la rhizosphère, par plusieurs mécanismes notamment par la production des acides organiques, par l'assimilation différentielle des anions et des cations, par leur influence sur les activités microbiennes (Hinsinger et al., 2003). Ces mécanismes portent essentiellement sur la libération des ions $\mathrm{H}^{+}$ou $\mathrm{OH}^{-}$dans la solution du sol par les racines des plantes pour maintenir le $\mathrm{pH}$ intracellulaire (Hinsinger et al., 2003). Par exemple, les espèces végétales qui utilisent l'azote sous forme ammoniacale vont entrainer une diminution $\mathrm{du} \mathrm{pH}$ du sol tandis que celles utilisant le nitrate comme source d'azote vont provoquer une augmentation du $\mathrm{pH}$ du sol. Les valeurs élevées du $\mathrm{pH}_{\mathrm{KCl}}$ sous les graminées pérennes signifient qu'elles joueraient un meilleur rôle de tampon pour le pH du sol que les graminées annuelles. De ce fait, on peut penser que ces graminées pérennes, en interaction avec d'autres facteurs du milieu ou pas, atténueraient les variations de $\mathrm{pH}$ des sols que les graminées annuelles. La capacité tampon du sol est fortement corrélée à la capacité d'échange cationique (Xu et al., 2012). Les graminées pérennes pourraient augmenter cette capacité d'échange cationique (CEC) par leur capacité à accumuler de la matière organique et à limiter le lessivage (anions, cations et argiles) grâce à leur système racinaire dense. L'hypothèse de l'accumulation de la matière organique sous la graminée est vérifiée pour $A$. ascinodis mais pas pour A. gayanus.

Ce travail, basé sur des observations de terrain, ne permet pas de séparer parfaitement les effets des graminées sur le sol d'un possible effet de l'hétérogénéité du sol sur la distribution des graminées. Cependant, la comparaison à une échelle spatiale très fine, des résultats obtenus avec les échantillons de sol prélevés, sur le même type de sol, sous les touffes de graminées et sur le sol nu (sol situé entre les touffes de graminées) ont conduits à interpréter ces résultats comme l'impact d'une 
modification du sol (pH, CEC, matière organique du sol, etc.) par la présence des graminées à travers des mécanismes qui restent à préciser. Pour répondre strictement à cette question il faudrait réaliser une expérience en conditions contrôlées où les différentes espèces sont cultivées dans les mêmes conditions (par exemple une expérience en pots contenant tous le même sol homogénéisé).

Les teneurs élevées en carbone, en azote total et en phosphore assimilable obtenues sous la pérenne A. ascinodis signifient qu'elle pourrait participer au maintien et/ou à l'amélioration du stockage de ces éléments que les autres espèces. Plusieurs études ont montré une augmentation des teneurs en carbone total et en azote total sous les jachères à $A$. ascinodis (Somé et al., 2007). Pour ces auteurs, les graminées pérennes ( $A$. ascinodis et $A$. gayanus) auraient des propriétés leur permettant d'améliorer le statut organique des sols que les graminées annuelles.

Les teneurs en ammonium et en nitrates des sols sont plus élevées sous les graminées pérennes $A$. ascinodis et $A$. gayanus que sous les graminées annuelles (A. pseudapricus et $L$. togoensis) et le sol nu. Ces résultats confirment le fait que ces deux types de graminées influencent différemment le cycle de l'azote. Les graminées pérennes contrôlent mieux le recyclage de l'azote et limiteraient les pertes (Yé et al., 2015). On peut supposer que cette différence est due à des quantités d'azote globalement plus élevées sous les graminées pérennes. Cette hypothèse est vérifiée pour $A$. ascinodis mais pas pour $A$. gayanus qui ne présente pas de teneurs en azote total significativement différentes des autres.

La respiration basale est plus importante sous les graminées pérennes que sous le sol nu et les graminées annuelles. L'activité respirométrique reflète l'activité microbienne globale du sol. Son importance dans un sol indique une bonne activité minéralisatrice totale et dépend de la qualité du substrat organique du sol. Ainsi, les valeurs élevées de la respiration basale sous les graminées pérennes $A$. ascinodis et $A$. gayanus indiquent la présence d'un pool microbien actif et d'une richesse plus élevée en matière organique facilement minéralisable sous ces dernières que sous les graminées annuelles et le sol nu. Des résultats similaires ont été aussi obtenus sous ces deux graminées pérennes dans une jachère artificielle à $A$. ascinodis et A. gayanus (Somé et al., 2007).

La biomasse microbienne n'est ici pas liée au «type biologique» de graminées. En revanche, elle semble être liée à la teneur en carbone total du sol car la plus importante valeur est observée sous l'espèce pérenne $A$. ascinodis où l'on observe également les teneurs les plus élevées en carbone total et en azote total. Cette corrélation positive entre la biomasse microbienne et la teneur en carbone du sol a aussi été observée par d'autres auteurs (Groffman et al., 2001).

Les résultats pris dans leur ensemble montrent que les activités enzymatiques sont plus importantes sous les graminées pérennes que sous les graminées annuelles. Ces activités enzymatiques du sol sont le plus souvent liées à la teneur en matière organique et l'azote total du sol (Aon et Colaneri, 2001). Cette explication générale peut convenir pour nos deux Graminées pérennes même si le $\mathrm{N}$ et le $\mathrm{C}$ total n'augmente que significativement pour A. ascinodis.

Les résultats montrent que le pâturage n'a pas d'effet significatif sur les paramètres chimiques étudiés. Ceci serait dû à la courte durée de l'expérimentation. En effet, certaines études ont montré qu'à court terme le pâturage n'a pas d'effet significatif sur les teneurs en carbone total et en azote total même si on peut observer une légère diminution de la matière organique du sol (Silveira et al., 2013). Cependant, à long terme, le pâturage diminue les teneurs en carbone et en azote totaux du sol (Han et al., 2008; Prieto et al., 2011). Par ailleurs, il semblerait qu'une forte intensité de pâturage stimule la nitrification, et que la dénitrification augmente sous une faible 
intensité de pâturage (Le Roux et al., 2003) ce qui devrait à long terme affecter les teneurs en nitrate et en ammonium du sol. Une augmentation du pH et de la densité du sol a aussi été observée avec l'augmentation de l'intensité du pâturage due principalement aux apports d'urine et au piétinement (Prieto et al., 2011).

Ces résultats montrent que le pâturage influence significativement la respiration basale des sols, mais pas les autres paramètres biologiques. Elle est en effet plus élevée dans les parcelles protégées, indiquant une bonne activité de minéralisation microbienne et un pool organique minéralisable plus élevé dans ces dernières que dans les parcelles non protégées. Ceci est comparable aux résultats de certaines études qui ont montré que la défoliation des espèces végétales diminuait l'activité respirométrique des sols (Shahzad et al., 2012). La respiration basale est liée à la disponibilité des fractions organiques labiles (Wang et al., 2003) et est stimulée par un apport de matières organiques fraîches facilement décomposables (Prévost-Bouré et al., 2010). De ce fait, son augmentation dans les parcelles protégées serait due à plus de matières organiques biodisponibles dans celles-ci que dans les parcelles non protégées. Contrairement à ces résultats, certaines études, en prairie humide pâturée, ont observé que le pâturage entraine une augmentation de la biomasse microbienne (Prieto et al., 2011) et une stimulation de l'activité minéralisatrice des sols pouvant conduire à des pertes de carbone (Klumpp et al., 2009). L'augmentation de la biomasse microbienne serait due à l'apport de nutriments minéraux sous forme d'urine et de matières organiques labiles sous forme de fèces, entrainant ainsi le développement des microorganismes dans les sites intensément pâturés (Prieto et al., 2011). Le pâturage modifie également les communautés et les activités microbiennes (Patra et al., 2005) qui pourraient entrainer des effets négatifs ou positifs sur la minéralisation du carbone et de l'azote (Bardgett et Wardle, 2003). En outre, le pâturage stimulerait le recyclage (minéralisation) de l'azote (Le Roux et al., 2003; Klumpp et al., 2009). D'autres activités microbiennes impliquées dans le cycle des nutriments (carbone, azote, phosphore) semblent être aussi affectées par le pâturage (Le Roux et al., 2003; Patra et al., 2005; Prieto et al., 2011). Par exemple, la nitrification (Le Roux et al., 2003) et les activités de la bêta-glucosidase, de la phosphatase et de la protéase (Prieto et al., 2011) augmentent avec l'intensité de pâturage. La dénitrification augmente sous une faible intensité de pâturage (Le Roux et al., 2003). Les résultats des différentes études ci-dessus évoquées montraient que les effets du pâturage sur la végétation et sur les caractéristiques des sols dépendent de la durée et de l'intensité de pâturage. Ceci dit, les résultats de la présente étude pourraient, en partie, s'expliquer par la courte durée de l'expérimentation mais aussi par l'intensité de pâturage (non contrôlée au cours de l'étude). A cela s'ajoute le feu qui est aussi un facteur pouvant perturber le fonctionnement des écosystèmes pâturés. En effet, dans les savanes, le feu, tout comme le pâturage, est un facteur important qui affecte la composition spécifique et la structure de la végétation (Fournier et al., 2001). Le feu qui, dans le site où cette étude a été menée, brûle annuellement la biomasse aérienne à la fois des exclos et des parcelles contrôles pourrait masquer certains effets du bétail (Holdo et al., 2007).

\section{Conclusion}

Tous ces résultats montrent que les effets du pâturage sur le recyclage des nutriments relèvent d'interactions assez complexes. On a obtenu en 18 mois peu d'effets sur le fonctionnement (paramètres chimiques et biologiques) du sol mais de nombreux effets sur les biomasses de plantes. Cela suggère, sur le pas de temps de cette étude, que les effets du bétail sur le recyclage des nutriments et la durabilité des pâturages passent plus par des modifications de la communauté végétale que par un impact 
direct du bétail sur le sol. Cette hypothèse reste bien sûr à tester en maintenant les exclos pendant plusieurs années de suite. De ce fait, la mise en place d'un dispositif comportant des parcelles protégées contre les feux et le bétail s'avère nécessaire afin de déterminer les effets isolés de ces deux facteurs qui font partie intégrante du fonctionnement de cet écosystème.

\section{CONFLIT D'INTERETS}

Les auteurs déclarent sur l'honneur l'absence de tout conflit d'intérêt.

\section{CONTRIBUTIONS DES AUTEURS}

Tous les auteurs ont contribué à l'œuvre présentée. L. Yé a réalisé les travaux de terrain et la plupart des mesures au laboratoire. L. Yé, S. Barot, H. B. Nacro, D. Masse et J-C. Lata ont conçu l'échantillonnage, interprété les résultats et ont été impliqués dans la rédaction du manuscrit. Ils ont tous approuvé la version finale du manuscrit.

\section{REMERCIEMENTS}

Lambiénou Yé a été financé par le Programme AIRD / BSTD et EC2CO pour son doctorat. Nous remercions les laboratoires LEMSAT et LAMA de l'IRD à Dakar pour les différentes analyses des sols. Nous remercions également Roger Kissou et Saïbou Nignan pour leur aide lors de la caractérisation du sol et l'installation des parcelles.

\section{REFERENCES}

Adam G, Duncan H. 2001. Development of a sensitive and rapid method for the measurement of total microbial activity using fluorescein diacetate (FDA) in a range of soils. Soil Biology \& Biochemistry, 33: 943-951. www.elsevier.com/locate/soilbio.

Amato M, Ladd JN. 1988. Essay for microbial biomass based on ninhydrin- reactive nitrogen in extracts of fumigated soils. Soil Biology \& Biochemistry, 20:107-114.
Aon MA, Colaneri AC. 2001. II. Temporal and spatial evolution of enzymatic activities and physico-chemical properties in an agricultural soil. Applied Soil Ecology, 18: 255-270. www.elsevier.com/locate/apsoil

Bardgett RD et Wardle DA. 2003. Herbivoremediated linkages between aboveground and belowground communities. Ecology, 84: 2258-2268. Speacial Feature, Footnote 1, p. 2256.

Boudsocq S, Lata JC, Mathieu J, Abbadie L, Barot S. 2009. Modelling approach to analyze the effects of nitrification inhibition on primary production. Functional Ecology, 23: 220-230. DOI: 10.1111/j.1365-2435.2008.01476.x

Bremner JM. 1965. Nitrogen availability indexes. Agronomy, 9: 1324-1345.

Crawley MJ. 2007. The R book. Wiley, Chichester, UK. 942 p.

Dabin B. 1967. Application des dosages automatiques à l'analyse des sols. Cahier ORSTOM, 5: 257-286.

Fournier A, Floret C, Gnahoua G-M. 2001. Végétation des jachères et succession post-culturale en Afrique tropicale. In $L a$ Jachère en Afrique Tropicale, Floret C, Pontanier R (eds). John Libbey Eurotext 4;123-168.

Gonnety JT, Assémien EFL, Guéi AM, N'dri AA, Djina Y, Koné AK, Tondoh JE. 2012. Effect of land-use types on soil enzymatic activities and chemical properties in semi-deciduous forest areas of Central-west Côte d'Ivoire. Biotechnologie Agronomie Société et Environnement, 16: 478-485.

Groffman PM, McDowell WH, Myers JC, Merriam JL. 2001. Soil microbial biomass and activity in tropical riparian forests. Soil Biology \& Biochemistry, 33: 1339-1348. www.elsevier.com/locate/soilbio

Guitian R, Bardgett RD. 2000. Plant and soil microbial responses to defoliation in temperate semi-natural grassland. Plant 
and Soil 220: 271-277. Kluwer Academic Publishers.

Han G, Hao X, Zhao M, Wang M, Ellert BH, Willms W, Wang M. 2008. Effect of grazing intensity on carbon and nitrogen in soil and vegetation in a meadow steppe in Inner Mongolia. Agriculture, Ecosystems and Environment, 125: 21-32. DOI:10.1016/j.agee.2007.11.009

Hayano K. 1973. A method for determination of beta-glucosidase activity in soil. Soil Science and Plant Nature, 19: 103-108.

Hinsinger P, Plassard C, Tang C, Jaillard B. 2003. Origins of root-mediated $\mathrm{pH}$ changes in the rhizophere and their responses to environmental constraints: a review. Plant and Soil, 248: 43-59.

Holdo RM, Holt RD, Coughenour MB, Ritchie ME. 2007. Plant productivity and soil nitrogen as a function of grazing, migration and fire in an African savanna. Journal of Ecology, 95: 115-128. DOI: 10.1111/j.1365-2745.2006.01192.x

Klumpp K, Fontaine S, Attard E, Le Roux X, Gleixner G, Soussana J-F. 2009. Grazing triggers soil carbon loss by altering plant roots and their control on soil microbial community. Journal of Ecology, 97: 876885. DOI: $\quad 10.1111 / \mathrm{j} .1365$ 2745.2009.01549.x

Le Roux X, Bardy M, Loiseau P, Louault F. 2003. Stimulation of soil nitrification and denitrification by grazing in grasslands: do changes in plant species composition matter? Oecologia, 137: 417-425. DOI 10.1007/s00442-003-1367-4

Oumorou M, Aboh BA, Babatounde S, Houinato M, Sinsin B. 2010. Valeur pastorale, productivité et connaissances endogènes de l'effet de l'invasion, par Hyptis suaveolens L. Poit., des pâturages naturels en zone soudano-guinéenne (Bénin). Int. J. Biol. Chem. Sci., 4(4): 1262-1277.

http://indexmedicus.afro.who.int

Patra AK, Abbadie L, Clays-Josserand A, Degrange V, Grayston SJ, Loiseau P, Louault F, Mahmood S, Nazaret S,
Philippot L, Poly F, Prosser JI, Richaume A, Le Roux X. 2005. Effects of grazing on microbial functional groups involved in soil $\mathrm{N}$ dynamics. Ecological Monographs, 75: 65-80.

Prévost-Bouré NC, Soudani K, Damesin C, Berveiller D, Lata JC, et Dufrêne E. 2010. Increase in aboveground fresh litter quantity over-stimulates soil respiration in a temperate deciduous forest. Applied Soil Ecology, 46: 26-34. DOI:10.1016/j.apsoil.2010.11.006

Prieto LH, Bertiller MB, Carrera AL, Olivera NL. 2011. Soil enzyme and microbial activities in a grazing ecosystem of Patagonian Monte, Argentina. Geoderma, 162: 281-287. DOI: 10.1016/j.geoderma.2011.02.011

Rakotoarimanana V, Gondard H, Ranaivoarivelo N, Carriere S. 2008. Influence du pâturage sur la diversité floristique, la production et la qualité fourragères d'une savane des Hautes Terres malgaches (région de Fianarantsoa). Sécheresse, 19(1): 39-46. DOI: $10.1684 / \mathrm{sec} .2008 .0120$

Sawadogo L, Tiveau D, Nygard R. 2005. Influence of selective tree cutting, livestock and prescribed fire on herbaceous biomass in the savannah woodlands of Burkina Faso, West Africa.

Agriculture, Ecosystems and Environment, 105: 335-345. DOI: 10.1016/j.agee.2004.02.004

Shahzad T, Chenu C, Repinçay C, Mougin C, Ollier J-L, Fontaine S. 2012. Plant clipping decelerates the mineralization of recalcitrant soil organic matter under multiple grassland species. Soil Biology \& Biochemistry, 51: 73-80. DOI: 10.1016/j.soilbio.2012.04.014

Silveira ML, Liu K, Sollenberger LE, Follett RF, Vendramini JMB. 2013. Short-term effects of grazing intensity and nitrogen fertilization on soil organic carbon pools under perennial grass pasture in the southeastern USA. Soil Biology \& Biochemistry, $\quad$ 58: $\quad 42-49$. 
http://dx.doi.org/10.1016/j.soilbio.2012.1 1.003

Somé NA, Traoré K, Traoré O, Tassembedo M. 2007. Potentiel des jachères artificielles à Andropogon spp. dans l'amélioration des propriétés chimiques et biologiques des sols en zone soudanienne (Burkina Faso). Biotechnologie Agronomie Société Environement, 11(3): 245-252.

Tabatabai MA, Bremner JM. 1969. Use of pnitrophneyl phosphate for assay of soil phosphatase activity. Soil Biology \& Biochemistry, 1: 301-307.

Toko I, Sinsin B. 2011. Facteurs déterminant la variabilité spatiale de la biomasse herbacée dans la zone soudano-guinéenne du Bénin. Int. J. Biol. Chem. Sci., 5(3): 930-943. http://indexmedicus.afro.who.int

Wang WJ, Dalal RC, Moody PW, Smith CJ. 2003. Relationships of soil respiration to microbial biomass, substrate availability and clay content. Soil Biology \& Biochemistry, 35: 273-284. www.elsevier.com/locate/soilbio

Wittig R, Hahn-Hadjali K, Krohmer J, Müller J, Sieglstetter R. 2002. La végétation actuelle des savanes du Burkina Faso et du Bénin. Sa signification pour l'homme et la modification de celle-ci par l'homme (aperçu des résultats d'un projet de recherche durée des années). Etudes Flo. Vég. Burkina Faso, 7: 03-16.
Xu R-K, Zhao A-Z, Yuan J-H, Jiang J. 2012. $\mathrm{pH}$ buffering capacity of acid from tropical and subtropical regions of China as influenced by incorporation of crop straw biochars. Journal of Soils and Sediments, 12: 494-502. DOI 10.1007/s11368-012-0483-3

Yaméogo G, Kiéma A, Yélémou B, Ouédraogo L. 2013. Caractéristiques des ressources fourragères herbacées des pâturages naturels du terroir de Vipalogo (Burkina Faso). Int. J. Biol. Chem. Sci., 7(5): 2078-2091. DOI: http://dx.doi.org/10.4314/ijbcs.v7i5.25

Yé L, Abbadie L, Bardoux G, Lata JC, Nacro HB, Masse D, de Parseval H, Barot S. 2015. Contrasting impacts of grass species on nitrogen cycling in a grazed Sudanian savanna. Acta Oecologica, 63: 8-15. http://dx.doi.org/10.1016/j.actao.2015.01. 002

Zoffoun AG, Aboh AB, Adjolohoun S, Houinato M, Sinsin B. 2013. Effet de l'âge et de l'intensité de pâture sur le développement des touffes et la production de biomasse de Panicum maximum var. $\mathrm{C} 1$ dans les pâturages artificiels en zone soudanienne et subéquatoriale. Int. J. Biol. Chem. Sci., 7(3): $\quad 1168-1179 . \quad$ DOI: http://dx.doi.org/10.4314/ijbcs.v7i3.23 